
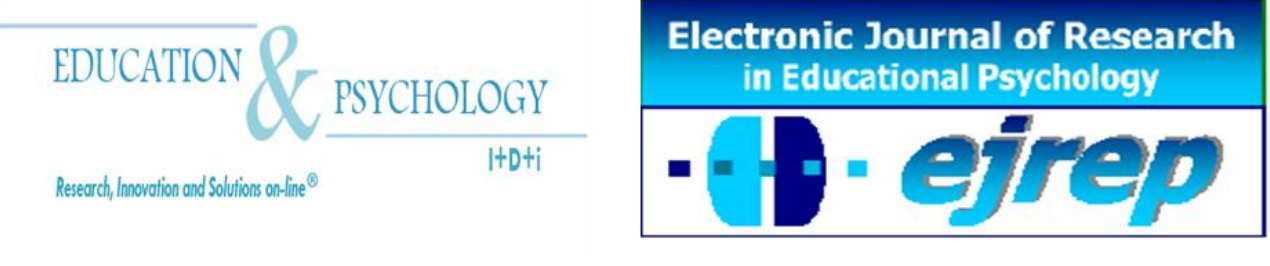

Editorial EOS

\title{
Acoso Escolar en la Comunidad de Extremadura vs. Informe Español del Defensor del Pueblo (2006)
}

\section{Benito León del Barco ${ }^{1}$, Elena Felipe Castaño ${ }^{1}$, Teresa Gómez Carroza, ${ }^{1}$ Víctor López Ramos ${ }^{1}$}

${ }^{1}$ Departamento de Psicología y Antropología, Universidad de Extremadura

\section{España}

Correspondencia: Benito León del Barco. Facultad de Formación del Profesorado. Avda de la Universidad S/N 10071 Cáceres. España. E-mail: bleon@unex.es

(C) Education \& Psychology I+D+i and Editorial EOS (Spain) 


\section{Resumen}

Introducción. Con este trabajo pretendemos realizar un estudio epidemiológico sobre el maltrato escolar que permita conocer la situación real en los centros de la Comunidad de Extremadura. Entre los objetivos que nos hemos propuesto destacamos: 1. Analizar la prevalencia de las diferentes modalidades de maltrato y comparar los resultados con los encontrados por el trabajo "Violencia Escolar: El maltrato entre iguales en la Educación Secundaria" del Defensor del Pueblo (2006) 2. Conocer el papel que juegan el género, curso y la titularidad pública o privada del centro en la incidencia y modalidad del acoso escolar.

Método. El número de participantes se determinó a partir del número de alumnos matriculados en secundaria en Extremadura durante el curso 2008-2009, considerando un error muestral de $3 \%$ y un nivel de confianza de 95,5\%. Finalmente, el número de participantes fue de 1700 estudiantes de Educación Secundaria de Extremadura, pertenecientes a 22 centros públicos y privados-concertados. Hemos utilizado el Cuestionario del Defensor de Pueblo-Unicef (2006), es un cuestionario autoadministrado que analiza la incidencia del acoso escolar desde tres perspectivas: observador, agresor y víctima.

Resultados. Podemos apreciar porcentajes, ligeramente, más altos en los centros ESO de Extremadura. Se han encontrado diferencias significativas (Prueba Chi Cuadrado) debidas al género, el curso y titularidad del centro, tanto desde el punto de vista de la víctima como el del agresor, en diferentes modalidades de maltrato escolar.

Discusión y Conclusión. Todas las modalidades de maltrato tienen lugar en todos los centros estudiados de secundaria en la Comunidad de Extremadura. Sin embargo, existe una relación inversa entre "gravedad" del maltrato y frecuencia. Los chicos se ven implicados con mayor frecuencia en el fenómeno bullying, tanto como agresores y víctimas, a excepción de la maledicencia más asociada a las chicas. Existe una tendencia general a que las víctimas sean alumnos de primeros cursos, especialmente, primero de la ESO y los agresores sean, fundamentalmente, alumnos de $4^{\circ}$ de la ESO. Por ultimo, los centros privados/concertados están más involucrados en el maltrato escolar en las modalidades de exclusión social y agresión verbal. Nuestros resultados obtenidos durante el curso 2009-10 coinciden con las investigaciones realizadas por el Defensor del Pueblo $(1999,2006)$

Palabras Clave: Acoso escolar, agresor, víctima, estudiantes, educación secundaria. 


\title{
School bullying in the Extremadura region vs. the Ombudsman's Report (2006)
}

\begin{abstract}
Introduction. In this paper we conduct an epidemiological study on school bullying as to show the real situation in the centers of the Community of Extremadura. Among the objectives we have set include: 1 . To analyze the prevalence of different forms of abuse and compare the results with those found by the job "School Violence: Bullying in Secondary Education" of the Ombudsman (2006) 2. To know the role of gender, course and public or private institution in the incidence and type of bullying.

Method. The number of participants was determined from the number of students enrolled in secondary school in Extremadura during 2008-2009, considering a sampling error of 3\% and a confidence level of $95.5 \%$. Finally, the number of participants was 1700 students of Secondary Education of Extremadura, from 22 public and private-arranged. We used the questionnaire of the Ombudsman-Unicef (2006), is a questionnaire that examines the impact of bullying from three perspectives: observer, perpetrator and victim.

Results. We appreciate percentages slightly higher in the centers of Extremadura ESO. We found significant differences (Chi Square Test) due to gender, grade and type of school, from the viewpoint of the victim and the aggressor, in different forms of school bullying.

Discussion and Conclusion. All forms of abuse occur in all studied secondary schools in the Community of Extremadura. However, there is an inverse relationship between the "seriousness" of abuse and frequency. The boys are more frequently involved in the bullying, both as perpetrators and victims, with the exception of slander more associated with girls. There is a general tendency for the victims are students from primary grades, especially, the first of the ESO and the perpetrators are mainly students of $4^{\circ}$ ESO. Finally, private schools/concluded are more involved in school bullying in patterns of social exclusion and verbal aggression. Our results obtained during the year 2009-10 are consistent with research as realized by the Ombudsman $(1999,2006)$
\end{abstract}

Keywords: School bullying, bullying, victim, students, secondary education. 


\section{Introducción}

Las primeras investigaciones sobre maltrato escolar, conocido como victimización y por los vocablos ingleses bullying y mobbing, surgen en Noruega y Suecia y son desarrolladas por Olweus $(1978,1999)$. Estas investigaciones ponen de manifiesto una incidencia de un 10\% del matonismo a nivel escolar, lo que lleva al Ministerio de Educación Noruego a desarrollar a nivel nacional una campaña de estudio y prevención. Para Olweus (1983), pionero en el estudio de este fenómeno, el maltrato o abuso entre iguales es una conducta de persecución física y/o psicológica que realiza el alumno o alumna contra otro, al que elige como víctima de repetidos ataques. Actualmente, las formas tradicionales de maltrato entre iguales han ido cambiando con el transcurrir del tiempo, apareciendo manifestaciones más específicas denominadas cyberbullying (Smith, Mahdavi, Carvalho y Tippet, 2006; Ortega, Calmaestra y Mora-Merchan, 2008) que se sirven de las nuevas tecnologías de la información y de la comunicación para acosar con ensañamiento a la víctima.

Con posterioridad a las investigaciones de Olweus $(1978,1999)$, surgen al final de la década de los 80 los primeros estudios en Inglaterra (Whitney y Smith, 1993). En Escocia fue Mellor (1990) quien realizó el primer trabajo sobre la incidencia del abuso entre iguales y en Irlanda tenemos a Byrne (1994) y O’Moore y Hillery (1989). En Alemania los estudios se inician en los años 90, en concreto Funk (1997). En cuanto a las investigaciones en países mediterráneos, se han realizado en Italia y Portugal. El estudio pionero en Italia es de 1996 por parte de Genta, Menesini, Fonzi, Costabile y Smith. En Portugal hay que hacer referencia a los trabajos de Pereira, Mendoza, Neto, Almeida, Valente y Smith (1996) y Almeida (1999).

En nuestro país, aunque son muchas las investigaciones realizadas en diferentes comunidades, pioneras como Andalucía y Madrid (Ortega y Angulo, 1998; Viera, Fernández y Quevedo, 1989), son tres los estudios relevantes a nivel nacional que se han llevado a cabo: Defensor del Pueblo 1999- 2006, Estudio Cisneros X “Violencia y Acoso Escolar” en 2005 (Piñuel y Oñate, 2007) e Informe del Centro Reina Sofía "Violencia entre Compañeros en la Escuela" en 2005 elaborado por Serrano e Iborra. Como conclusiones generales de todos estos trabajos, destacaríamos: que el maltrato entre iguales es un fenómeno que sucede en todos los países que se ha estudiado y que provoca en las víctimas efectos claramente negativos: des- 
censo de la autoestima, estados de ansiedad e incluso cuadros depresivos, lo que dificulta su integración en el medio escolar y el desarrollo normal de los aprendizajes.

En general el efecto más notable del maltrato entre iguales es la aparición de desórdenes emocionales como estrés, ansiedad y depresión, además de problemas para integrarse en el grupo de iguales debido al aislamiento y a la exclusión social. En Noruega Olweus (1993) realizó un estudio durante siete años y encontró una relación entre el hecho de ser víctima de maltrato escolar a los 15-16 años y un mayor nivel de depresión a los 23 años. En Estados Unidos Kochenderfer-Ladd y Wardrop (2001) encontraron relaciones entre ser victimizados y la soledad y nivel de satisfacción social en 388 niños estudiados desde la guardería hasta tercer curso de primaria. En España con una muestra de 196 profesores de secundaria y 145 estudiantes de universitarios, Van der Meulen, Soriano, Granizo, Del Barrio, Korn y Schäfer (2003) ponen de manifiesto la relación entre maltrato y autoestima. Investigaciones como la realizada por Estévez, Martínez y Musitu (2006) sobre un total de 965 adolescentes con edades comprendidas entre los 11 y 16 años muestran que los adolescentes victimizados en la escuela se perciben a sí mismos más negativamente en los ámbitos social y emocional de la autoestima.

La preocupación social que suscita la violencia en los contextos sociales ha dado lugar a numerosas intervenciones y acciones en diferentes países y en muy diferentes ámbitos (Martín, Fernández, Andrés, Del Barrio y Echeita, 2003). La mayoría de las investigaciones se centran en el ámbito escolar y pretenden dos objetivos: en un primer lugar la investigación epidemiológica de la gravedad del fenómeno en cada contexto o entorno cultural (país), mediante cuestionarios u otros procedimientos de recogida de información, en segundo lugar una labor de intervención dirigida hacia todos los elementos implicados, individuales (agresor y víctima), familiares, escolares (organización y políticas educativas) y sociales (marco legal y políticas sociales), tanto para detener el proceso como para su prevención. Para Garaigordobil y Oñederra (2010) se ha pasado en los últimos años de no intervenir a realizar cantidad de estudios y campañas preventivas en los centros. Entre las intervenciones centradas en el alumnado destacan las propuestas de ayuda entre iguales, que Cowie y Wallace (2000) clasifican en dos grupos: las que enfatizan el apoyo emocional y las que enfatizan la educación entre iguales como el aprendizaje cooperativo, éste es una metodología útil para reducir las conductas de acoso en el aula. 
Con este trabajo pretendemos realizar un estudio epidemiológico sobre el maltrato escolar que permita conocer la situación real en los centros de la Comunidad de Extremadura, de los que aún no disponemos de datos fiables. Entre los objetivos que nos hemos propuesto destacamos: 1. Analizar la prevalencia de las diferentes modalidades de maltrato y comparar los resultados con los encontrados por el trabajo "Violencia Escolar: El maltrato entre iguales en la Educación Secundaria" del Defensor del Pueblo (2006) 2. Conocer el papel que juegan el género, el curso, la edad y la titularidad pública o privada del centro en la incidencia y modalidad del acoso escolar.

\section{Método}

\section{Participantes}

La selección de los estudiantes se realizó mediante un muestreo polietápico estratificado por conglomerados y selección aleatoria de los grupos en los centros que disponían de varias líneas en los cursos $1^{\circ}, 2^{\circ}, 3^{\circ}$ y $4^{\circ}$ de la ESO. Los estratos que se consideraron fueron los Centros de Recursos de Profesores (CPRs) que dividen en 18 zonas geográficas la Comunidad de Extremadura, cada CPR tiene asignados unos determinados Centros de Secundaria (IES e IESO). El muestreo por conglomerado consistió en seleccionar al azar dos centros de cada CPR. Algunos centros declinaron la participación en el estudio.

El número de participantes se determinó a partir del número de alumnos matriculados en secundaria en Extremadura durante el curso 2008-2009, considerando un error muestral de $3 \%$ y un nivel de confianza de 95,5\%. Finalmente, el número de participantes fue de 1708 estudiantes de Educación Secundaria de Extremadura, pertenecientes a 22 centros, 17 públicos y 5 privados-concertados. Respecto a la distribución por género, 864 son mujeres frente a 840 varones. De primero de la ESO participaron 474 alumnos, 452 de segundo de la ESO, 400 de tercero y 382 de cuarto de la ESO.

\section{Instrumentos}

Hemos utilizado el Cuestionario del Defensor de Pueblo-Unicef (2006): es un cuestionario autoadministrado que analiza la incidencia del acoso escolar desde tres perspectivas: observador, agresor y víctima. Las preguntas incluyen trece conductas de acoso que se clasifican en seis modalidades de maltrato. La ocurrencia del maltrato es evaluada en una escala 
ordinal de frecuencia con cuatro categorías: nunca, a veces, a menudo y siempre. También, evalúa algunas características de los agresores como curso, género y los lugares en los que ocurre cada tipo de acoso.

\section{Procedimiento}

Una vez seleccionados al azar los centros educativos y los cursos, nos pusimos en contacto con los directores de los centros y los tutores de los cursos para informales del objeto de nuestro trabajo y solicitarles su colaboración. Posteriormente, en una misma sesión los alumnos cumplimentaron siguiendo los criterios de voluntariedad y anonimato el Cuestionario del Defensor de Pueblo-Unicef (2006). El tiempo invertido osciló entre 30 y 40 minutos y previamente se había solicitado su consentimiento de forma verbal. Participaron dos entrevistadores por curso y estuvieron siempre disponibles para resolver cualquier consulta de los alumnos.

\section{Resultados}

En primer lugar presentaremos los resultados desde la perspectiva víctima, después agresor y, finamente, observador. Para las perspectivas víctima y agresor se estudiará el papel de las variables: género, curso y titularidad centro.

\section{Víctimas}

En la figura 1 presentamos los porcentajes de alumnos que declaran ser víctimas de las distintas situaciones de acoso escolar y los comparamos con los porcentajes del Informe Defensor del Pueblo-Unicef (2006). Podemos apreciar porcentajes, ligeramente, más altos en los centros ESO de Extremadura. 


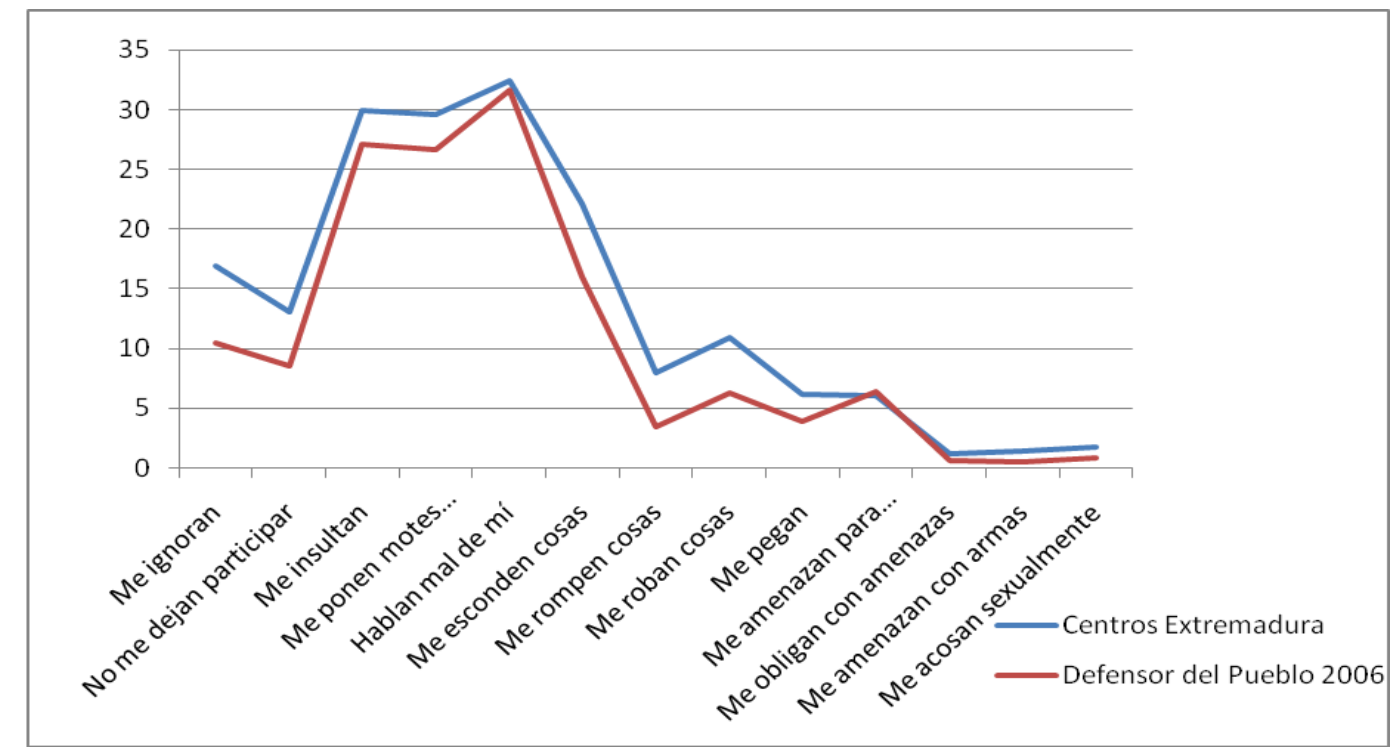

Figura 1. Porcentajes de víctimas, comparativa Centros ESO de Extremadura vs. Informe Defensor del Pueblo (2006).

No obstante, existe una tendencia común en ambos estudios, son las conductas de agresión verbal: el insulto, la burla y el poner motes como las más frecuentes seguidas de las conductas de exclusión social. Nos llama la atención desde el punto de vista investigador encontrar la misma línea en los dos estudios.

Como se observa en la tabla 1, el género de los participantes está relacionado con la incidencia como víctima en algunos de los tipos de maltrato. Hay más chicos que chicas que se reconocen como víctimas en conductas de agresión verbal como: recibir insultos y motes. En el caso de "hablar mal de mí" y "me roban cosas" ocurre lo contrario, siendo significativamente más numeroso el porcentaje de chicas que de chicos. Por otro lado, los chicos reconocen que les pegan más y reciben más agresiones de amenazas y chantajes.

Tabla 1. Porcentajes de víctimas en las distintas situaciones de acoso escolar según el género y resultados prueba Chi Cuadrado.

\begin{tabular}{llccc}
\hline \multicolumn{2}{c}{ Tipos de malos tratos } & $\begin{array}{c}\text { Chica } \\
\mathbf{n = 8 6 4}\end{array}$ & $\begin{array}{c}\text { Chico } \\
\mathbf{n = 8 4 0}\end{array}$ & $\begin{array}{c}\text { Prueba Chi Cua- } \\
\text { drado }\end{array}$ \\
\hline \multirow{3}{*}{ Exclusión Social } & Me ignoran & 16,5 & 17,4 & $\chi^{2}=0,237 ; \mathrm{p}<0,626$ \\
\cline { 2 - 5 } & No me dejan participar & 11,5 & 14,7 & $\chi^{2}=3,752 ; \mathrm{p}<0,053$ \\
\hline \multirow{2}{*}{ Agresión verbal } & Me insultan & 25,2 & 34,8 & $\chi^{2}=18,550 ; \mathbf{p}<\mathbf{0 , 0 0 0}$ \\
\cline { 2 - 5 } & Me ponen motes ofensivos & 25,8 & 33,5 & $\chi^{2}=12,051 ; \mathbf{p}<\mathbf{0 , 0 0 1}$ \\
\cline { 2 - 5 } & Hablan mal de mí & 34,9 & 29,8 & $\chi^{2}=5,021 ; \mathbf{p}<\mathbf{0 , 0 2 5}$ \\
\hline & Me esconden cosas & 22,5 & 21,6 & $\chi^{2}=0,191 ; \mathrm{p}<0,662$ \\
\hline
\end{tabular}




\begin{tabular}{llccc}
\hline $\begin{array}{l}\text { Agresión Física } \\
\text { indirecta }\end{array}$ & Me rompen cosas & 7,2 & 9,0 & $\chi^{2}=1,806 ; \mathbf{p}<0,179$ \\
\cline { 2 - 5 } & Me roban cosas & 12,6 & 9,2 & $\chi^{2}=4,855 ; \mathbf{p}<\mathbf{0 , 0 2 8}$ \\
\hline Agresión F. directa & Me pegan & 5,1 & 7,5 & $\chi^{2}=3,991 ; \mathbf{p}<\mathbf{0 , 0 4 6}$ \\
\hline \multirow{2}{*}{$\begin{array}{l}\text { Amenazas y chan- } \\
\text { tajes }\end{array}$} & Me amenazan para miedo & 4,4 & 7,8 & $\chi^{2}=8,562 ; \mathbf{p}<\mathbf{0 , 0 0 3}$ \\
\cline { 2 - 5 } & Me obligan con amenazas & 0,7 & 1,8 & $\chi^{2}=4,079 ; \mathbf{p}<\mathbf{0 , 0 4 3}$ \\
\cline { 2 - 5 } & Me amenazan con armas & 0,5 & 2,1 & $\chi^{2}=9,295 ; \mathbf{p}<\mathbf{0 , 0 0 2}$ \\
\hline Acoso sexual & Me acosan sexualmente & 0,8 & 2,4 & $\chi^{2}=6,595 ; \mathbf{p}<\mathbf{0 , 0 1 0}$ \\
\hline
\end{tabular}

Respecto a la variable curso, podemos observar en la tabla 2 que existen diferencias significativas en algunos tipos de maltrato. Los alumnos de primer curso de la ESO reciben más insultos y motes que sus compañeros de cursos superiores, les pegan y les amenazan. Los alumnos de últimos cursos de la ESO reconocen que se habla mal de ellos y les roban en un número significativamente mayor de veces que los de primeros cursos.

Tabla 2. Porcentajes de víctimas en las distintas situaciones de acoso escolar según el curso y resultados prueba Chi Cuadrado.

\begin{tabular}{|c|c|c|c|c|c|}
\hline Tipos de malos tratos & $\begin{array}{l}\text { 19ESO } \\
\mathrm{n}=474\end{array}$ & $\begin{array}{l}2 \circ E S O \\
\mathrm{n}=452\end{array}$ & $\begin{array}{l}\text { 3ESO } \\
\mathrm{n}=400\end{array}$ & $\begin{array}{l}\text { 4.ESO } \\
\mathrm{n}=382 \\
\end{array}$ & $\begin{array}{c}\text { Prueba } \\
\text { Chi Cuadrado } \\
\end{array}$ \\
\hline Me ignoran & 21 & 14,8 & 15,8 & 15,7 & $\chi^{2}=7,790 ; \quad \mathrm{p}<0,051$ \\
\hline No me dejan participar & 16,2 & 13,1 & 10,8 & 11,8 & $\chi^{2}=6,367 ; \quad \mathrm{p}<0,095$ \\
\hline Me insultan & 38,6 & 29,3 & 27,6 & 22,8 & $\chi^{2}=27,223 ; \mathbf{p}<\mathbf{0 , 0 0 0}$ \\
\hline Me ponen motes ofensivos & 36,5 & 25 & 32,1 & 24,1 & $\chi^{2}=22,146 ; \mathbf{p}<\mathbf{0 , 0 0 0}$ \\
\hline Hablan mal de mí & 33,3 & 26,6 & 35,1 & 35,2 & $\chi^{2}=9,653 ; \quad \mathbf{p}<\mathbf{0 , 0 2 2}$ \\
\hline Me esconden cosas & 19,8 & 19,4 & 25,3 & 24,6 & $\chi^{2}=7,133 ; \quad p<0,068$ \\
\hline Me rompen cosas & 8,7 & 6,9 & 6,3 & 10,7 & $\chi^{2}=1,806 ; \quad \mathrm{p}<0,179$ \\
\hline Me roban cosas & 9,1 & 9,1 & 10,0 & 16,2 & $\chi^{2}=14,549 ; \mathbf{p}<\mathbf{0 , 0 0 2}$ \\
\hline Me pegan & 11,3 & 6,7 & 5,0 & 1,0 & $\chi^{2}=38,738 ; \mathbf{p}<\mathbf{0 , 0 0 0}$ \\
\hline Me amenazan para miedo & 11,0 & 5,8 & 4,5 & 2,1 & $\chi^{2}=32,608 ; \mathbf{p}<\mathbf{0 , 0 0 0}$ \\
\hline Me obligan con amenazas & 2,3 & 1,3 & 0,8 & 0,3 & $\chi^{2}=8,483 ; \quad \mathbf{p}<\mathbf{0 , 0 3 7}$ \\
\hline Me amenazan con armas & 1,5 & 1,3 & 2,0 & 0,3 & $\chi^{2}=4,918 ; \quad \mathrm{p}<0,178$ \\
\hline Me acosan sexualmente & 2,3 & 1,5 & 1,8 & 0,5 & $\chi^{2}=4,538 ; \mathrm{p}<0,209$ \\
\hline
\end{tabular}

Finalmente, con relación a la variable titularidad del centro se observa en la tabla 3 una diferencia significativa en la conducta "no me dejan participar" y "hablan mal de mí" a favor de los centros privados/concertados. 
Tabla 3. Porcentajes de víctimas en las distintas situaciones de acoso escolar según la titularidad del centro y resultados prueba Chi Cuadrado.

\begin{tabular}{lllcc}
\hline \multirow{2}{*}{ Tipos de malos tratos } & $\begin{array}{c}\text { Centro } \\
\text { Público } \\
\mathbf{n = 1 2 2 7}\end{array}$ & $\begin{array}{c}\text { Privado/ } \\
\text { Concertado } \\
\mathbf{n = 4 7 7}\end{array}$ & $\begin{array}{c}\text { Prueba Chi Cua- } \\
\text { drado }\end{array}$ \\
\hline \multirow{3}{*}{ Exclusión Social } & Me ignoran & 16,2 & 18,9 & $\chi^{2}=1,712 ; \mathrm{p}<0,191$ \\
\cline { 2 - 5 } & No me dejan participar & 12,1 & 15,7 & $\chi^{2}=3,999 ; \mathbf{p}<\mathbf{0 , 0 4 6}$ \\
\hline \multirow{3}{*}{ Agresión verbal } & Me insultan & 29,3 & 31,7 & $\chi^{2}=0,954 ; \mathrm{p}<0,329$ \\
\cline { 2 - 5 } & Me ponen motes ofensivos & 29,4 & 30,2 & $\chi^{2}=0,954 ; \mathrm{p}<0,755$ \\
\cline { 2 - 5 } & Hablan mal de mí & 30,9 & 36,1 & $\chi^{2}=4,239 ; \mathbf{p}<\mathbf{0 , 0 4 0}$ \\
\hline \multirow{3}{*}{$\begin{array}{l}\text { Agresión Física } \\
\text { indirecta }\end{array}$} & Me esconden cosas & 22,6 & 20,6 & $\chi^{2}=0,846 ; \mathrm{p}<0,358$ \\
\cline { 2 - 5 } & Me rompen cosas & 7,3 & 10,1 & $\chi^{2}=3,417 ; \mathrm{p}<0,065$ \\
\cline { 2 - 5 } & Me roban cosas & 11,2 & 10,1 & $\chi^{2}=0,495 ; \mathrm{p}<0,482$ \\
\hline Agresión F. directa & Me pegan & 6,6 & 5,5 & $\chi^{2}=0,779 ; \mathrm{p}<0,377$ \\
\hline \multirow{2}{*}{$\begin{array}{l}\text { Amenazas y chan- } \\
\text { tajes }\end{array}$} & Me amenazan para miedo & 5,9 & 6,5 & $\chi^{2}=0,181 ; \mathrm{p}<0,671$ \\
\cline { 2 - 5 } & Me obligan con amenazas & 1,2 & 1,3 & $\chi^{2}=0,003 ; \mathrm{p}<0,954$ \\
\cline { 2 - 5 } & Me amenazan con armas & 1,4 & 1,0 & $\chi^{2}=0,308 ; \mathrm{p}<0,579$ \\
\hline Acoso sexual & Me acosan sexualmente & 1,5 & 1,9 & $\chi^{2}=0,388 ; \mathrm{p}<0,533$ \\
\hline
\end{tabular}

\section{Agresores/as}

En la figura 2 presentamos los porcentajes de alumnos que reconocen ser agresores y los comparamos con los porcentajes del Informe Defensor del Pueblo-Unicef (2006). Podemos apreciar porcentajes más altos en los centros ESO de nuestra comunidad. 


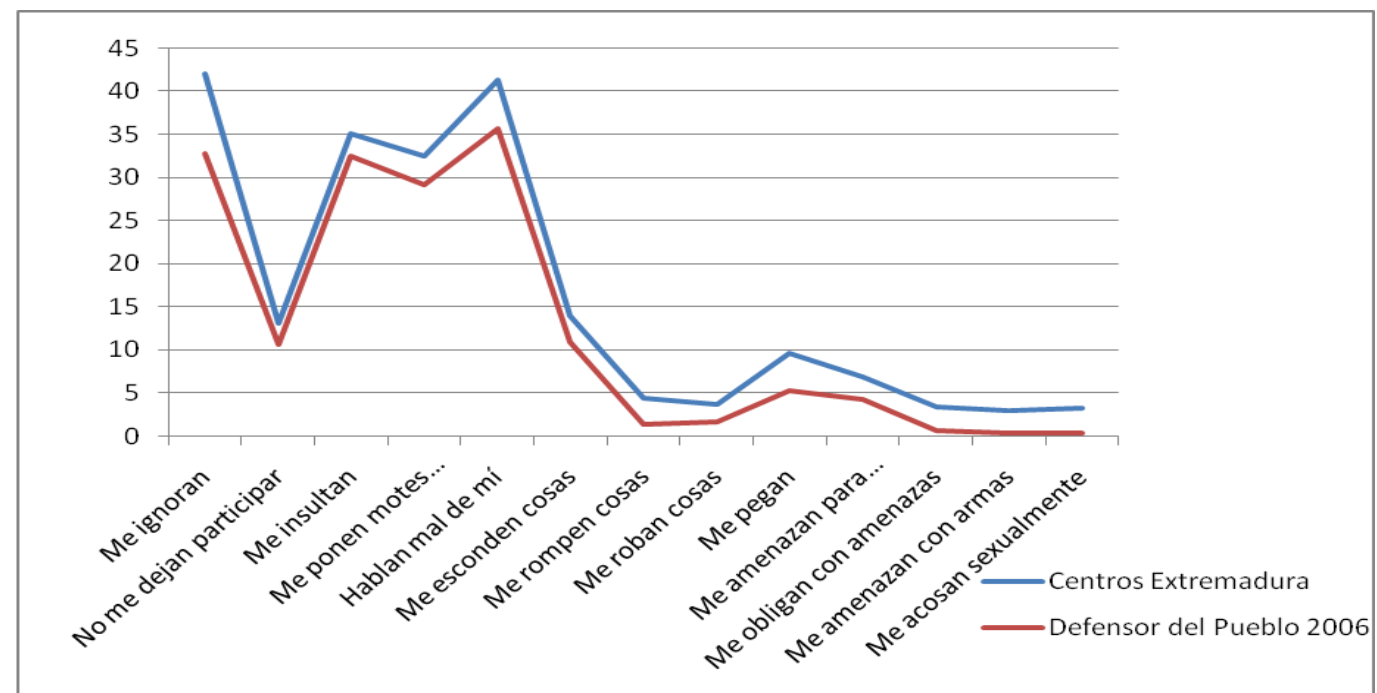

Figura 2. Porcentajes de agresores/as, comparativa Centros ESO de Extremadura vs. Informe Defensor del Pueblo (2006)

Se repite el mismo patrón en lo que se refiere a una mayor frecuencia en conductas de exclusión social y agresión verbal. Además, como ocurría en la perspectiva víctima las líneas que representan los porcentajes de forma gráfica coinciden de nuevo en los dos estudios.

En lo que respecta a la influencia del género sobre la incidencia de agresores/as en las distintas modalidades de maltrato, en la tabla 4 podemos apreciar que los varones se declaran más agresores que las chicas en las conductas de agresión verbal "insulto" y "pongo motes ofensivo". Esta situación se invierte en la conducta "hablo mal de alguien", donde las chicas superan a los chicos. Se observan, asimismo, más diferencias con respecto al género a favor de los varones en conductas como "escondo cosas", "pego" y "obligo con amenazas".

Tabla 4. Porcentajes de agresores/as en las distintas situaciones de acoso escolar según el género y resultados prueba Chi Cuadrado.

\begin{tabular}{llccc}
\hline \multicolumn{2}{c}{ Tipos de malos tratos } & $\begin{array}{c}\text { Chica } \\
\mathbf{n = 8 6 4}\end{array}$ & $\begin{array}{c}\text { Chico } \\
\mathbf{n = 8 4 0}\end{array}$ & $\begin{array}{c}\text { Prueba Chi Cuadra- } \\
\text { do }\end{array}$ \\
\hline \multirow{3}{*}{ Exclusión Social } & Ignoro & 41,7 & 42,4 & $\chi^{2}=0,082 ; \mathrm{p}<0,774$ \\
\cline { 2 - 5 } & No dejo participar & 11,7 & 14,4 & $\chi^{2}=2,743 ; \mathrm{p}<0,098$ \\
\hline \multirow{2}{*}{ Agresión verbal } & Insulto & 32,4 & 37,7 & $\chi^{2}=5,328 ; \mathbf{p}<\mathbf{0 , 0 2 1}$ \\
\cline { 2 - 5 } & Pongo motes ofensivos & 30,0 & 35,0 & $\chi^{2}=4,843 ; \mathbf{p}<\mathbf{0 , 0 2 8}$ \\
\cline { 2 - 5 } & Hablo mal de los demás & 47,7 & 34,7 & $\chi^{2}=29,358 ; \mathbf{p}<\mathbf{0 , 0 0 0}$ \\
\hline \multirow{2}{*}{$\begin{array}{l}\text { Agresión Física } \\
\text { indirecta }\end{array}$} & Escondo cosas & 12,4 & 15,8 & $\chi^{2}=3,882 ; \mathbf{p}<\mathbf{0 , 0 4 9}$ \\
\cline { 2 - 5 } & Rompo cosas & 3,8 & 4,7 & $\chi^{2}=1,806 ; \mathrm{p}<0,398$ \\
\cline { 2 - 5 } & Robo cosas & 3,5 & 3,8 & $\chi^{2}=0,139 ; \mathrm{p}<0,710$ \\
\hline
\end{tabular}




\begin{tabular}{lllll}
\hline Agresión F. directa & Pego & 7,5 & 11,7 & $\chi^{2}=8,507 ; \mathbf{p}<\mathbf{0 , 0 0 4}$ \\
\hline \multirow{3}{*}{$\begin{array}{l}\text { Amenazas y chan- } \\
\text { tajes }\end{array}$} & Amenazo para meter miedo & 6,0 & 7,6 & $\chi^{2}=1,704 ; \mathrm{p}<0,192$ \\
\cline { 2 - 5 } & Obligo con amenazas & 2,3 & 4,4 & $\chi^{2}=5,779 ; \mathbf{p}<\mathbf{0 , 0 1 6}$ \\
\cline { 2 - 5 } & Amenazo con armas & 2,2 & 3,8 & $\chi^{2}=3,789 ; \mathrm{p}<0,052$ \\
\hline Acoso sexual & Acoso sexualmente & 2,9 & 3,6 & $\chi^{2}=0,613 ; \mathrm{p}<0,434$ \\
\hline
\end{tabular}

Respecto al curso, podemos ver en la tabla 5 que también causa diferencias significativas en conductas de exclusión social y agresión verbal. Los últimos cursos de la ESO y especialmente los de $4^{\circ}$ curso se declaran más agresores en las modalidades "no dejo participar", "pongo motes ofensivos", "hablo mal de los demás" y "escondo cosas".

Tabla 5. Porcentajes de agresores/as en las distintas situaciones de acoso escolar según el curso y resultados prueba Chi Cuadrado.

\begin{tabular}{|c|c|c|c|c|c|}
\hline Tipos de malos tratos & $\begin{array}{l}\text { 19ESO } \\
n=474\end{array}$ & $\begin{array}{l}2^{\circ} \text { ESSO } \\
\mathrm{n}=452\end{array}$ & $\begin{array}{l}3^{\circ} E S O \\
n=400\end{array}$ & $\begin{array}{l}4^{\circ} \text { ESO } \\
\mathrm{n}=382\end{array}$ & $\begin{array}{c}\text { Prueba } \\
\text { Chi Cuadrado }\end{array}$ \\
\hline Ignoro & 40,0 & 42,8 & 41,5 & 44,5 & $\chi^{2}=1,872 ; \quad \mathrm{p}<0,599$ \\
\hline No dejo participar & 11,3 & 10,6 & 13,1 & 18,1 & $\chi^{2}=11,933 ; \mathbf{p}<\mathbf{0 , 0 0 8}$ \\
\hline Insulto & 32,0 & 32,8 & 39,2 & 37,0 & $\chi^{2}=6,574 ; \quad p<0,087$ \\
\hline Pongo motes ofensivos & 32,5 & 27,1 & 32,9 & 38,3 & $\chi^{2}=12,026 ; \mathbf{p}<\mathbf{0 , 0 0 7}$ \\
\hline Hablo mal de los demás & 36,8 & 36,8 & 46,6 & 46,7 & $\chi^{2}=16,961 ; \quad \mathbf{p}<\mathbf{0 , 0 0 1}$ \\
\hline Escondo cosas & 9,2 & 14,2 & 15,3 & 18,6 & $\chi^{2}=16,097 ; \quad \mathbf{p}<\mathbf{0 , 0 0 1}$ \\
\hline Rompo cosas & 3,4 & 4,2 & 3,0 & 6,5 & $\chi^{2}=7,210 ; \quad \mathrm{p}<0,065$ \\
\hline Robo cosas & 2,6 & 4,9 & 2,5 & 4,7 & $\chi^{2}=6,149 ; \quad \mathrm{p}<0,105$ \\
\hline Pego & 10,3 & 7,3 & 10,6 & 10,5 & $\chi^{2}=3,748 ; \quad p<0,290$ \\
\hline Amenazo para meter miedo & 6,6 & 7,5 & 7,3 & 5,8 & $\chi^{2}=1,201 ; p<0,753$ \\
\hline Obligo con amenazas & 3,0 & 3,3 & 3,8 & 3,4 & $\chi^{2}=0,383 ; \quad \mathrm{p}<0,944$ \\
\hline Amenazo con armas & 2,6 & 3,5 & 1,8 & 4,2 & $\chi^{2}=4,718 ; \quad \mathrm{p}<0,194$ \\
\hline Acoso sexualmente & 2,8 & 3,8 & 2,5 & 3,9 & $\chi^{2}=1,975 ; \quad p<0,578$ \\
\hline
\end{tabular}

Por último, la titularidad del centro, también, influye en la prevalencia de los agresores en las modalidades de exclusión social y agresión verbal. Podemos apreciar en la tabla 6 que los alumnos de centros privados/concertados se declaran más agresores que los alumnos de centros públicos en las conductas de exclusión social y agresión verbal. 
Tabla 6. Porcentajes de agresores/as en las distintas situaciones de acoso escolar según la titularidad del centro y resultados prueba Chi Cuadrado.

\begin{tabular}{|c|c|c|c|c|}
\hline \multicolumn{2}{|c|}{ Tipos de malos tratos } & \multirow{2}{*}{ 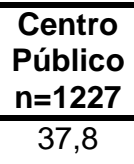 } & \multirow{2}{*}{$\begin{array}{c}\begin{array}{c}\text { Privado/ } \\
\text { Concertado } \\
\mathbf{n}=477\end{array} \\
53,3\end{array}$} & \multirow{2}{*}{$\begin{array}{c}\begin{array}{c}\text { Prueba Chi Cua- } \\
\text { drado }\end{array} \\
\chi^{2}=33,66 ; \quad \text { p }<0,000\end{array}$} \\
\hline & Ignoro & & & \\
\hline Exclusión Social & No dejo participar & 11,3 & 17,7 & $\chi^{2}=12,33 ; \quad \mathbf{p}<\mathbf{0 , 0 0 0}$ \\
\hline \multirow{3}{*}{ Agresión verbal } & Insulto & 32,5 & 41,6 & $\chi^{2}=12,355 ; \mathbf{p}<\mathbf{0 , 0 0 0}$ \\
\hline & Pongo motes ofensivos & 29,4 & 40,4 & $\chi^{2}=19,11 ; \mathbf{p}<\mathbf{0 , 0 0 0}$ \\
\hline & Hablo mal de los demás & 37,7 & 50,5 & $\chi^{2}=23,12 ; \quad \mathbf{p}<\mathbf{0 , 0 0 0}$ \\
\hline \multirow{3}{*}{$\begin{array}{l}\text { Agresión Física } \\
\text { indirecta }\end{array}$} & Escondo cosas & 14,4 & 13,3 & $\chi^{2}=0,360 ; p<0,549$ \\
\hline & Rompo cosas & 4,5 & 3,6 & $\chi^{2}=0,697 ; \mathrm{p}<0,404$ \\
\hline & Robo cosas & 4,0 & 2,7 & $\chi^{2}=1,550 ; p<0,213$ \\
\hline Agresión F. directa & Pego & 9,4 & 10,1 & $\chi^{2}=0,186 ; p<0,667$ \\
\hline \multirow{3}{*}{$\begin{array}{l}\text { Amenazas y chan- } \\
\text { tajes }\end{array}$} & Amenazo para miedo & 6,4 & 8,0 & $\chi^{2}=1,415 ; p<0,234$ \\
\hline & Obligo con amenazas & 3,3 & 3,6 & $\chi^{2}=0,097 ; p<0,756$ \\
\hline & Amenazo con armas & 3,2 & 2,5 & $\chi^{2}=0,516 ; p<0,473$ \\
\hline Acoso sexual & Acoso sexualmente & 3,4 & 2,9 & $\chi^{2}=0,181 ; p<0,670$ \\
\hline
\end{tabular}

\section{Observadores/as}

En la figura 3 presentamos los porcentajes de alumnos testigos de las distintas situaciones de acoso escolar y los comparamos con los porcentajes del Informe Defensor del Pueblo-Unicef (2006). Podemos apreciar porcentajes, ligeramente, más altos en el informe del Defensor del Pueblo (2006). Este dato nos sorprende pues en el análisis de víctimas y agresores ocurría, exactamente, lo contrario. 


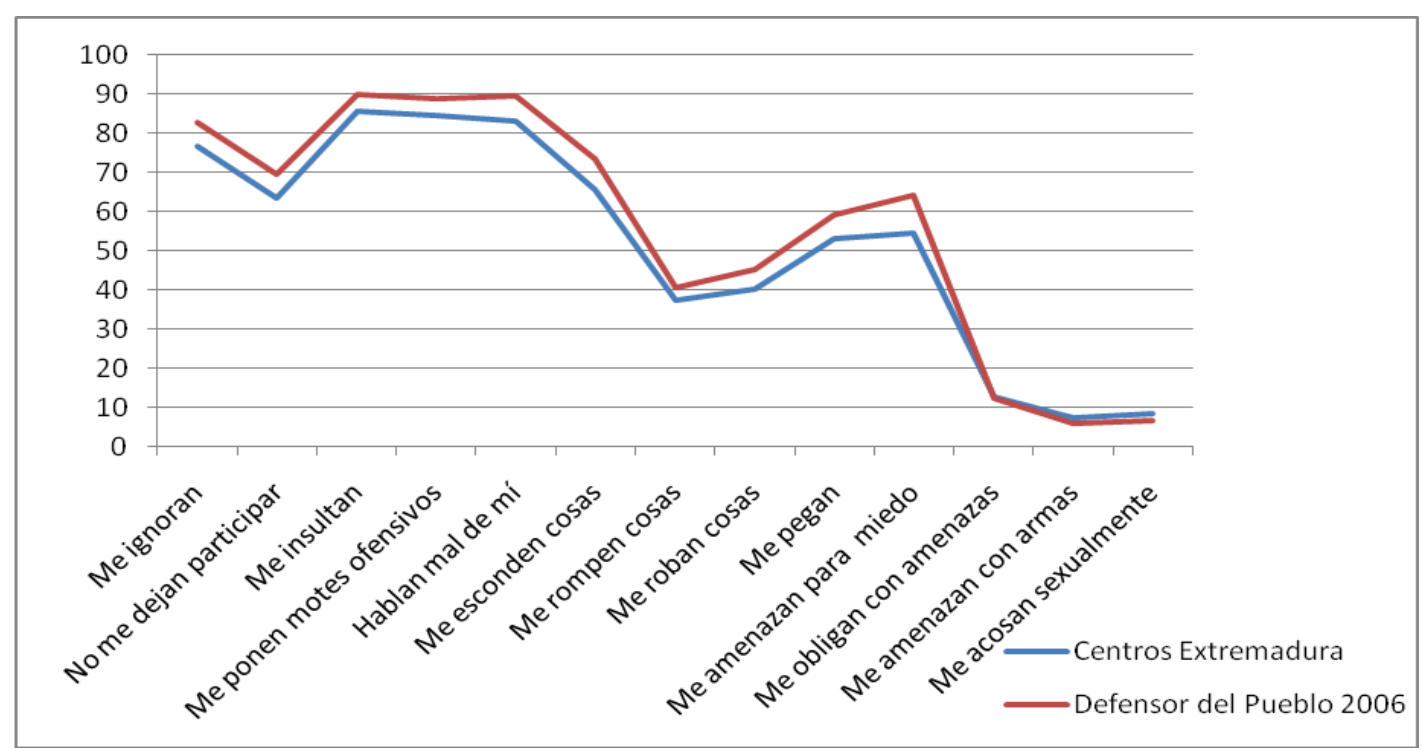

Figura 3. Porcentajes de observadores, comparativa Centros ESO de Extremadura vs. Informe Defensor del Pueblo (2006)

De nuevo coincide gráficamente la representación de los porcentajes de observadores de nuestro estudio y el realizado por el Defensor del Pueblo en el año 2006.

Por último, en la figura 4 hemos representado los porcentajes obtenidos en nuestra investigación desde la perspectiva víctima, agresor y observador. Observamos que los porcentajes de testigos son más elevados que los de víctimas y agresores, sin duda los espectadores de las distintas modalidades de maltrato deben ser muchos más que las víctimas y los agresores, un mismo caso de agresión puede ser observado por varios testigos. Analizando las tres perspectivas apreciamos unas pautas comunes: 1. las conductas más frecuentes corresponden a las agresiones de exclusión social y verbales, seguidas por un tipo de agresión física indirecta, "esconder cosas". 2. A un segundo nivel colocamos el resto de las conductas de agresión física indirecta con un máximo que corresponde a la agresión física directa "pegar". 3. Son las agresiones de amenazas y chantajes y el acoso sexual las menos frecuentes.

Los estudiantes que se reconocen agresores son menos que los que se declaran víctimas en la modalidad de agresiones físicas indirectas: "me esconden cosas", "me rompen cosas" y "me roban cosas". En el resto de modalidades son ligeramente más los estudiantes que se declaran agresores que los que se confiesan víctimas. Los agresores no parecen tener problemas para declararse autores de conductas de acoso, lo que sin duda debe preocuparnos desde el punto de vista de la prevención del maltrato escolar. 


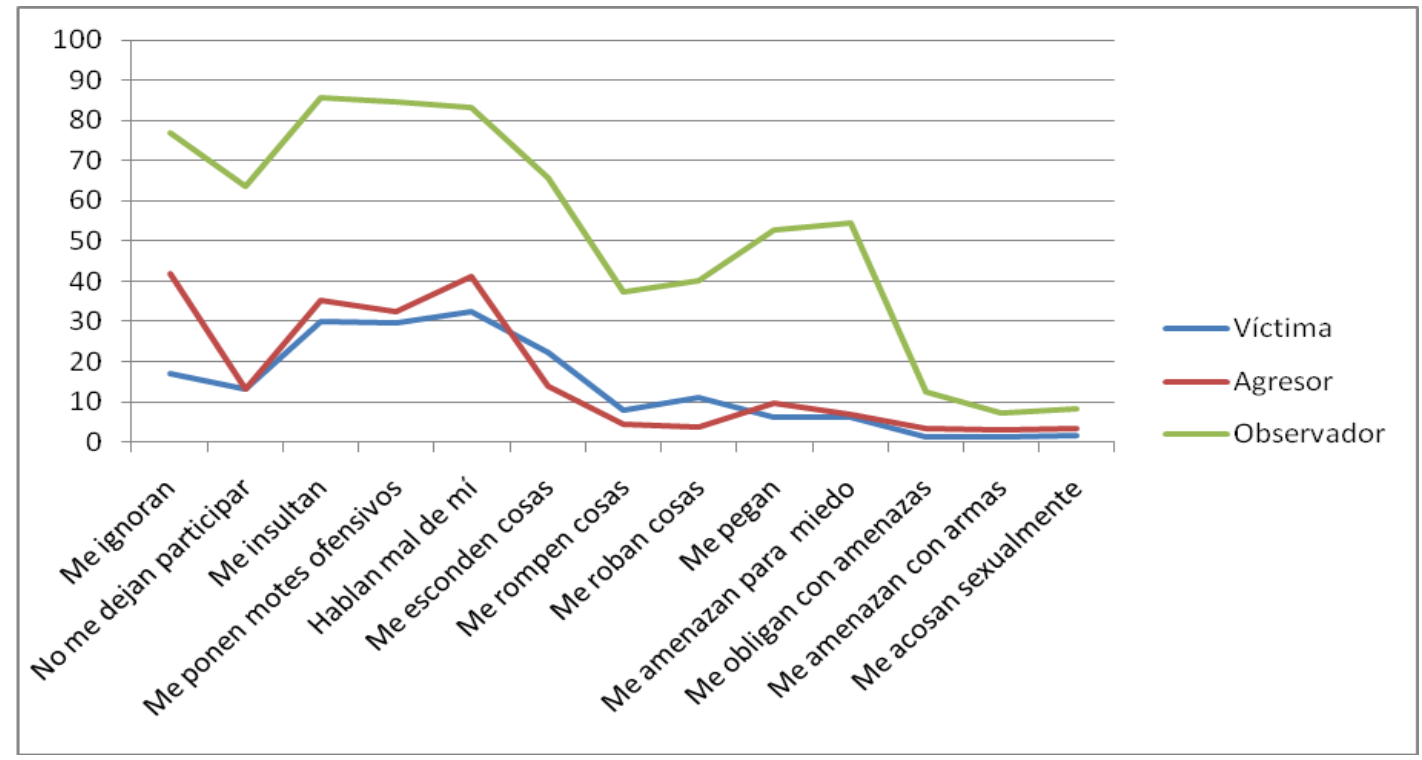

Figura 4. Comparativa porcentajes de víctimas, agresores/as y observadores de las diferentes modalidades de maltrato.

\section{Discusión y Conclusiones}

Con nuestro estudio pretendíamos analizar la prevalencia de las diferentes modalidades de maltrato, conocer el papel que juegan el género, el curso, la edad y la titularidad pública o privada del centro y estudiar características del acoso escolar: curso de quienes agreden y escenarios en los que se producen las acciones de maltrato.

Nuestros resultados ponen de manifiesto que todas las modalidades de maltrato tienen lugar en todos los centros estudiados de secundaria en la Comunidad de Extremadura. Este hecho coincide con otros estudios que evidencian que este fenómeno está presente en Educación Secundaria y con porcentajes considerables (Avilés y Monjas, 2005; Serrano e Iborra, 2005; Benítez y Justicia, 2006; Defensor del Pueblo, 2006). Sin embargo, existe una relación inversa entre "gravedad" del maltrato (acosar sexualmente, amenazar con armas y obligar mediante amenazas) y frecuencia, las conductas más frecuentes corresponden a las agresiones de exclusión social y verbales como el insulto, la burla y el poner motes, seguidas por un tipo de agresión física indirecta, "esconder cosas". A un segundo nivel colocamos el resto de las conductas de agresión física indirecta con un máximo que corresponde a la agresión física directa "pegar". Son las agresiones de mayor gravedad, las amenazas, chantajes y el acoso sexual las menos frecuentes. Conclusiones similares son referidas también en los informes del 
Defensor del Pueblo (1999, 2006). Esta relación inversa entre gravedad de maltrato e incidencia no debe hacernos pensar que este fenómeno es algo trivial, pues todas las formas de maltrato tienen consecuencias negativas sobre las víctimas. Como indica Ortega (1994), la situación de agresión destruye la autoestima y la confianza en sí mismo del escolar agredido, que puede llegar a estados depresivos o de permanente ansiedad que le harán más difícil su adaptación social y su rendimiento académico.

En nuestro estudio los porcentajes de testigos son más elevados que los de víctimas y agresores, los espectadores de las distintas modalidades de maltrato deben ser muchos más que las víctimas y los agresores, un mismo caso de agresión puede ser observado por varios testigos. Este hecho debería ser motivo de preocupación pues la exposición a la violencia, en todas sus manifestaciones (imitación de modelos agresivos, refuerzo operante directo de los actos agresivos, reforzamiento vicario a través del aprendizaje observacional) es un elemento fundamental mediante el cual niños y adolescentes aprenden conductas agresivas (Bandura, 1973; 1986). Existen muchas investigaciones que han demostrado la relación positiva entre exposición a la violencia en el contexto escolar y el desarrollo de conductas agresivas (Flannery, Wester y Singer, 2004; Garaigordobil y Oñederra, 2008; Cerezo, 2009).

Nuestros datos revelan que el género es una variable moduladora en la incidencia del maltrato, tanto desde el punto de vista de la víctima como el del agresor. Hay más chicos que chicas que se reconocen como víctimas en conductas de agresión verbal como: recibir insultos y motes. En el caso de "hablar mal de mí" ocurre lo contrario, siendo significativamente más numeroso el porcentaje de chicas que de chicos. Por otro lado, los chicos reconocen que les pegan más y reciben más agresiones de amenazas y chantajes. Este mismo patrón de diferencias significativas entre chicos y chicas se observa desde la perspectiva agresor. En general, nuestros resultados coinciden con otras investigaciones que indican que son los chicos los que se ven implicados con mayor frecuencia en el fenómeno bullying, tanto como agresores y víctimas (Solberg y Olweus, 2003; Tapper y Boulton, 2005; Cerezo y Ato, 2010), a excepción de la maledicencia más asociada a las chicas (Defensor del Pueblo, 2006). Para Sánchez y Cerezo (2010) el sexo es un factor de riesgo para la implicación en bullying, siendo este fenómeno preferentemente masculino. Algunas investigaciones encuentran que las mujeres tienen una mayor sensibilidad que los hombres. En general las mujeres son más expresivas que los hombres, autores como Lafferty (2004) y Tapia y Marsh (2006) entre otros, demostraron en sus investigaciones que las mujeres son más perceptivas, muestran mayor empatía y 
reconocen mejor las emociones ajenas, esto explicaría una interacción social más positiva con los demás y su menor involucración en el fenómeno maltrato escolar.

Con relación al curso, los alumnos de primer curso de la ESO reciben más insultos y motes que sus compañeros de cursos superiores, les pegan y les amenazan. Los alumnos de últimos cursos de la ESO reconocen que se habla mal de ellos y les roban en un número significativamente mayor de veces que los de primeros cursos. Desde la perspectiva agresor los últimos cursos de la ESO y especialmente los de $4^{\circ}$ curso se declaran más agresores en las modalidades "no dejo participar", "pongo motes ofensivos", "hablo mal de los demás" y "escondo cosas". La variable curso muestra una influencia significativa sobre los casos de maltrato escolar, existe una tendencia general a que las víctimas sean alumnos de primeros cursos, especialmente, primero de la ESO y los agresores sean, fundamentalmente, alumnos de $4^{\circ}$ de la ESO. Estos datos coinciden con otras investigaciones que afirman que es la edad de 11 a 13 donde se concentra un mayor número de víctimas y que los agresores son mayores que sus víctimas (Carney y Merrell, 2001; Seals y Young, 2003). Habría que determinar en estudios posteriores la influencia de los alumnos repetidores de curso sobre el fenómeno maltrato escolar, investigaciones como la de Cerezo (2006) señalan que la repetición es un factor de riesgo en las situaciones de maltrato, los alumnos repetidores que están escolarizados en cursos inferiores a los que les corresponden por edad actúan más frecuentemente como agresores.

La titularidad del centro, también, se ha convertido en nuestro estudio en una variable que influye en la prevalencia del maltrato. Así, desde la perspectiva víctima existen diferencias significativas en la conducta "no me dejan participar" y "hablan mal de mí" a favor de los centros privados/concertados. Desde la perspectiva agresor, los alumnos de centros privados/concertados se declaran más agresores que los alumnos de centros públicos en las conductas de exclusión social y agresión verbal. Como tendencia general los centros privados/concertados están más involucrados en el maltrato escolar en las modalidades de exclusión social y agresión verbal. Estos resultados coinciden con las investigaciones realizadas por el Defensor del Pueblo $(1999,2006)$ que señalan que la exclusión social y la maledicencia se ejerce y se sufre más en los centros privados o concertados que en los públicos.

A modo de reflexión nos planteamos algunas cuestiones que se pueden mejorar con relación a la metodología utilizada, en primer lugar la cumplimentación del cuestionario se realizó en el aula con todos los alumnos, algunas investigaciones destacan que este hecho y que 
víctimas y agresores estén juntos, puede afectar a la sinceridad de las respuestas. Por otro lado, el cuestionario utilizado nos ha permitico comparar los datos con los obtenidos en el informe del Defensor del Pueblo (2006), este cuestionario prima la investigación directa y recogida de información sobre cada tipo de maltrato en detrimento de características psicométricas del propio cuestionario (fiabilidad y validez).

Finalmente, añadir que para mejorar la convivencia y prevenir la violencia desde la escuela es imprescindible incorporar innovaciones educativas. Nos inclinamos por metodologías de enfoque socioafectivo y de estilo cooperativo. Como afirman Pérez y Garaigordobil (2004) la interacción entre iguales y en concreto, una dinámica socio-afectiva positiva en el aula, puede desempeñar un importante papel afectando no sólo a los procesos de socialización, sino también los procesos de desarrollo cognitivo, afectivo y emocional. El enfoque socioafectivo contribuye a la adquisición de competencias sociales, ya que la interacción constructiva con el grupo de iguales favorece e incrementa las habilidades sociales de los alumnos. Con relación al estilo cooperativo, Díaz-Aguado (2006) señala que las investigaciones llevadas a cabo en los últimos años permiten identificar al aprendizaje cooperativo en equipos heterogéneos como un procedimiento clave para adaptar la educación a los cambios sociales actuales, mejorando la convivencia escolar y la prevención de la violencia.

Pensamos en la utilidad de un trabajo en aprendizaje cooperativo en el aula para favorecer la convivencia y disminuir la violencia. Es innegable el hecho de que ser víctima escolar provoca alteraciones de comportamiento y trastornos afectivos emocionales. En general el efecto más notable del maltrato escolar es la aparición de desórdenes emocionales como estrés, ansiedad y depresión, además de problemas para integrarse en el grupo de iguales debido al aislamiento y a la exclusión social. El aprendizaje cooperativo es una metodología innovadora que puede ayudar a resolver el problema del maltrato entre iguales por abuso de poder en el ámbito escolar. El aprendizaje cooperativo es una herramienta eficaz para favorecer actitudes positivas ante las relaciones intergrupales (León, Felipe, Gozalo, Gómez y Latas, 2009). "Este método puede salvar las barreras a la amistad y a la interacción" (Slavin, 1983, p.136). 


\section{Referencias}

Almeida, A.M. (1999). Portugal. En P.K. Smith, Y. Morita, J. Junger-Tas, D. Olweus, R. Catalano, y P. Slee (Eds.), The nature of school bullying (pp. 174-187). London: Routhledge.

Avilés, J. y Monjas, I. (2005). Estudio de la incidencia de la intimidación y el maltrato entre iguales en la Educación Secundaria Obligatoria mediante el cuestionario CIMEI. Anales de Psicología, 21(1), 27-41.

Bandura, A. (1973). Aggression: A social learning analysis. Englewood Cliffs, NJ: Prentice Hall.

Bandura, A. (1986). Social foundations of thought and action. A social cognitive theory. Englewood Cliffs, NJ: Prentice Hall.

Benítez, J.L. y Justicia, F. (2006). El maltrato entre iguales: descripción del fenómeno. Electronic Journal of Research in Educational Psychology, 9 (4), 151-170.

Byrne, B. (1994). Bullying: A community approach. Dublin: Columbia Press.

Carney, A.G. y Merrell, K. W. (2001). Bullying in schools: Perspectives on understanding and preventing an international problem. School Psychology International, 22(3), 364382.

Cerezo, F. (2006). Violencia y victimización entre escolares. El bullying: Estrategias de identificación y elementos para la intervención a través del Test-Bull-S. Electronic Journal of Research in Educational Psychology, 9 (4), 333-352.

Cerezo, F. (2009). Bullying: análisis de la situación en las aulas españolas. International Journal of Psychology and Psychological Therapy, 9, 367-378.

Cerezo, F. y Ato, M. (2010). Social status, gender, classroom climate and bullying among adolescents pupils. Anales de Psicología, 26(1), 137-144.

Cowie, H. y Wallace, P. (2000). Peer support in action. London: Sage.

Defensor del Pueblo (1999). Informe Violencia escolar: El maltrato entre iguales en la Educación Secundaria Obligatoria. Madrid: Publicaciones de la Oficina del Defensor del Pueblo.

Defensor del Pueblo (2006). Violencia escolar: el maltrato entre iguales en la Educación Secundaria Obligatoria (1999-2006). Madrid: Publicaciones del Defensor del Pueblo.

Díaz-Aguado, M.J. (2006). Del acoso escolar a la cooperación en las aulas. Madrid: Pearson Educación. 
Estévez, E., Martínez, B. y Musitu, G. (2006). La autoestima en adolescentes agresores y víctimas en la escuela: la perspectiva multidimensional. Intervención Psicosocial, 15(2), 223-233.

Flannery, D.J., Wester, K.L. y Singer, M.I. (2004). Impact of exposure to violence in school on child and adolescent mental health and behavior. Journal of Community Psychology, $32,559-573$.

Funk, W. (1997). Violencia escolar en Alemania, estado del arte. Revista de Educación, 313, 53-78.

Garaigordobil, M. y Oñederra, J. A. (2008). Bullying: incidence of peer violence in the schools of the Autonomous Community of the Basque Country. International Journal of Psychology and Psychological Therapy, 8, 51-62.

Garaigordobil, M. y Oñederra, J. A. (2010). Un estudio comparativo de las conductas de acoso escolar percibidas por los directivos de los centros educativos y por los estudiantes de 10 a 16 años. En J. J. Gázquez y M.C. Pérez (Eds.), Investigación en convivencia escolar: variables relacionadas (pp. 97-104). Granada: GEU.

Genta, M.L., Menesini, E., Fonzi, A., Costabile, A. y Smith, P.K. (1996). Bullies and victims in schools in central and southern Italy. European Journal of Psychology of Education, $11,97-110$.

Kochenderfer-Ladd, B. y Wardrop. J.L. (2001) Cronicity and instability of children's peer victimization experiences as predictors of loneliness and social satisfaction trajectories. Child Development. 72, 134-151.

Lafferty, J (2004). The relationships betweengender, empathy and aggressive behavious among early adolescents. Dissertation Abstracts International: Section B: The Sciences and Engineering, 64, 6377B.

León, B., Felipe, E., Gozalo, M., Gómez, T. y Latas, C. (2009). Mejora de las actitudes de los escolares hacia los alumnos inmigrantes mediante el aprendizaje cooperativo. Revista de Psicología General y Aplicada, 9, 159-173.

Martín, E., Fernández, I., Andrés, S., Del Barrio, C. y Echeita, G. (2003). La intervención para la mejora de la convivencia en los centros educativos: modelos y ámbitos. Infancia y Aprendizaje, 26, 79-95.

Mellor, A. (1990). Spotlights 23. Bullying in Scottish Secondary Schools. Edinburgh: SCRE.

O’Moore, A.M. y Hillery, B. (1989). Bulling in Dublin schools. Iris Journal of Psychology, $10,426-441$. 
Olweus, D. (1978). Agression in the schools: Bullies and whipping hoys. Washington, D.C.: Hemisphere.

Olweus, D (1983). Low school achievement and agressive behaviour in adolescent boys. En D. Magnusson y V. Allen (Eds.), Human developrnent. An interactional perspective (pp. 353-365). Nueva York: Academic Press.

Olweus, D, (1993). Bullying at school: What we know and what we can do. Oxford: Blackwells. Trad. cast. Conductas de acoso y amenaza entre escolares. Madrid: Morata, 1998.

Olweus, D. (1999). Sweden. En P.K Smith, Y. Morita, J. Junger-Tas, D. Olweus, R. Catalano y P. Slee (Eds.), The nature of school bullying (pp. 7-28). London: Routledge.

Ortega, R. (1994). Violencia interpersonal en los centros educativos de enseñanza secundaria. Un estudio sobre el maltrato e intimidación entre compañeros. Revista de Educación, 304: 253-280.

Ortega, R. y Angulo, J. C. (1998). Violencia escolar. Su presencia en Institutos de Educación Secundaria en Andalucía. Estudios de Juventud, 42, 47-61.

Ortega, R., Calmaestra, J. y Mora, J. (2008) Cyberbullying. Internacional Journal of Psychology and Psychological Therapy, 8 (2), 183-192.

Pereira, B., Mendoza, D., Neto, C., Almeida, A., Valente, L. y Smith, E.K. (1996). Factors and figures of the first survey on bullying in Portuguese schools. Comunicación presentada en European Conference on Educational Research. Sevilla.

Pérez, J. I. y Garaigordobil, M. (2004). Socialización y personalidad a los seis años. Apuntes de Psicología, 22, 153-169.

Piñuel, I. y Oñate, A. (2007). Acoso y violencia escolar en España. Madrid: lieddi.

Sánchez, C. y Cerezo, F. (2010). Variables personales y sociales relacionadas con la dinámica bullying en escolares de Educación Primaria. Electronic Journal of Research in Educational Psychology, 8 (3), 1015-1032.

Seals, D. y Young, J. (2003). Bullying and victimization: Prevalence and relationship to gender, grade level, ethnicity, self-esteem and depression. Adolescence, 38(152), 735-747.

Serrano, A. y Iborra, I. (2005). Violencia entre compañeros en la escuela. Centro Reina Sofía para el Estudio de la Violencia.

Slavin, R.E. (1983). Cooperative Learning. New York: Longman. (Trad. cast.: La enseñanza y el método cooperativo. México. Edamex 1985). 
Smith, P.K., Mahdavi, J., Carvalho, C. y Tippett, N. (2006). An investigation into cyberbullying, its forms, awareness and impact, and the relationship between age and gender in cyberbullying. London: Anti-Bullying Alliance.

Solberg, M.E. y Olweus, D. (2003). Prevalence estimation of school bullying with the Olweus Bully/Victim questionnaire. Aggressive Behavior, 29(3), 239-268.

Tapia, M. y Marsh, G.E. (2006). The effects of sex and grade-point average on emocional intelligence. Psicothema, 18, 108-111.

Tapper, K. y Boulton, M.J. (2004). Sex differences in levels of physical, verbal, and indirect aggression in spanish adolescents. Aggessive Behavior, 30(2), 123-145.

Van der Meulen, K., Soriao, L., Granizo, L., Del Barrio, C., Korn, S. y Schäfer, M. (2003). Recordando el maltrato entre iguales en la escuela: consecuencias e influencia en la actuación del profesorado. Infancia y Aprendizaje, 26(1), 49-62.

Vieira, M., Fernández, I. y Quevedo, G. (1989). Violence, Bullying and counseling in the Iberian peninsula. En E. Roland y E. Munthe (Eds.), Bullyind an International Perspective (pp-35-52). Londres: David Fulton.

Whitney, I. y Smith, E.K. (1993). A survey of the nature and extent of bullying in junior/middle and secondary schools. Educational research 35, 3-25. 